www.nature.com/ijo

\title{
PREFACE
}

\section{Body composition: clinical implications and biological diversities}

\author{
$\mathrm{S} \mathrm{Zhu^{1 }}$ and Z Wang ${ }^{1,2}$ \\ ${ }^{1}$ Obesity and Body Composition Research Center, Zhejiang University School of Public Health, Hangzhou, China and \\ ${ }^{2}$ Obesity Research Center, St Luke's-Roosevelt Hospital, Columbia University, New York, NY, USA
}

International Journal of Obesity (2011) 35, S1-S3; doi:10.1038/ijo.2011.69

Human body composition study has a long history of more than 150 years. It focuses on the masses of various body components and their distribution, the quantitative relations among body components, the in vivo quantification of body components, and the quantitative changes in these components related to various intrinsic and extrinsic factors; the capacity to make these measurements has advanced very rapidly, with benefits both to the research scientists and to the populations of physicians and patients they serve. Human body composition studies are closely related to clinical medicine, sports science, geriatric medicine, nutrition, and so on, therefore having an important role in improving human health, as well as in characterizing the compositional nature and deviations in various health and disease states.

Since the 1960s, the 'modern era', when human body composition studies entered a new phase, the number of published reports has increased rapidly. By PubMed statistics, the number of papers with titles and/or keywords on body composition annually increased from about 20 in the $1950 \mathrm{~s}$ to 289 in the 1990s. Besides, in the first 6 years of the twentyfirst century, on average 1481 body composition papers were published annually worldwide.

Since 1986, the International Symposium on In Vivo Body Composition Studies has met every 3 years, serving as an international platform and stimulus for scholars around the world. The Ninth International Symposium will be held in May 2011 in Hangzhou, China, which is the first of the series to be held outside Europe and North America and so is a great symbolic event in body composition research in China. In all, 247 abstracts from 18 countries in Africa, Asia, Europe, North America, Oceania and South America have been

Correspondence: Dr S Zhu, Obesity and Body Composition Research Center, Zhejiang University School of Public Health, Hangzhou 310058, China or Dr Z Wang, Obesity Research Center, St Luke's-Roosevelt Hospital, Columbia University, 1090 Amsterdam Avenue, 14th Floor, New York, NY 10025, USA. E-mail: zsk@zju.edu.cn or zw28@columbia.edu received. This collection of abstracts is to be published as a supplement in the International Journal of Obesity. These abstracts are categorized into five sessions as shown below.

\section{Session I: Obesity, diabetes, the metabolic syndrome and body composition}

The prevalence of obesity has increased significantly worldwide. Both fat mass and fat distribution have been addressed in recent studies. Increased visceral adipose fat is strongly correlated with insulin resistance, the metabolic syndrome, diabetes and cardiovascular disease. Although all fat masses increase with age, subcutaneous fat mass is less, whereas visceral fat mass is larger, in men. Ethnicity also influences fat distribution. However, many of the studies have been on Asians transplanted to the United States and Europe; studies on the body composition of Asians living in Asia are still limited. The highest proportion of visceral adiposity in Chinese may be correlated with their high prevalence of insulin resistance in diabetes. It is probably inappropriate to apply the concepts and quantifications derived in Caucasians to Asian populations. A remarkable example for this is that whereas the body fat percent (\%BF) of Asians is 1-2 units lower than that of Caucasians, the body mass index (BMI) of Asians is $2-3 \%$ higher than that of Caucasians. This dilemma remains to be explained.

This session addresses the associations between body composition phenotypes and susceptibility to obesity, diabetes, cardiovascular disease and the metabolic syndrome. Two epidemiological studies, conducted by Huang et al. and He et al., respectively, explore the cut-off points of $\% \mathrm{BF}$ and waist circumference in predicting the risk of cardiovascular disease in Chinese people. Zhou et al. compared the predictive values of \%BF with BMI, and found a higher diagnostic value than BMI in men to predict both hypertension and arteriosclerosis. This large-sample study 
confirmed the limitations of BMI in predicting fat mass, in particular, at the individual level. Studies exploring the dynamic changes of body composition with age, and in selected disease states, are included in this session. Rhee et al. suggested that body composition may correlate with the risk factors for chronic diseases in obese, glucose-intolerant subjects. Adipose tissue-related molecular mechanisms in the pathological process of metabolic diseases, and ethnic variability in metabolic susceptibility to obesity and cardiovascular diseases, were comprehensively examined by others. Other than the deteriorations imposed by obesity on metabolic diseases, this session also addresses issues on the relationships of sarcopenia with the metabolic syndrome and insulin resistance.

\section{Session II: Other diseases and body composition}

Disease states, especially chronic disease states, may significantly alter human body composition. Measuring these body composition changes aids in making a correct diagnosis, and also influences the prognosis of the disease with treatment. Besides obesity, diabetes and the metabolic syndrome, other diseases have come into focus: osteoporosis, AIDS, certain cancers, hypertension, spinal cord injury, Alzheimer's disease, growth hormone deficiency, obstructive pulmonary disease, hyperthyroidism, non-alcoholic fatty liver and lupus.

In this session, several additional diseases have been studied. Wang et al. used $\mathrm{H}$ magnetic resonance spectroscopy (H-MRS) to quantify liver fat and found a positive relationship of blood C3 level with liver fat content. H-MRS can help elucidate the quantity of lipid in the liver, providing a basis for staging individuals at risk of disease. ${ }^{1}$ Yatsuya et al. found liver fat content to be associated with insulin resistance, independent of the degree of adiposity. There are approximately 9 million osteoporotic fractures each year, and the prevalence of osteoporosis is especially high in Asian regions. Dual-energy X-ray absorptiometry (DXA), quantitative ultrasound and quantitative computed tomography are used to evaluate bone structure and bone quality. Several studies related fat-free mass and fat mass with bone mass and quality. Xi et al. studied this association, and addressed the importance of fat distribution on bone mass and quality. Breast cancer is the most common invasive cancer among women in developed countries. ${ }^{2}$ Risks for second primary cancers and comorbidities, such as diabetes, cardiovascular disease and osteoporosis, are issues that need to be considered in long-term management of these women. ${ }^{3,4}$ By investigating the biochemical markers of bone formation and resorption as well as bone mineral density (BMD) in non-metastatic breast cancer (NMBC) in postmenopausal Egyptian women before and after adjuvant chemotherapy, Khalil et al. found adjuvant chemotherapy to be responsible for decreasing segmental and total BMD in
NMBC postmenopausal women. Metabolic disorders such as phenylketonuria are an inherited metabolic disorder of phenylalanine metabolism. Wilcox et al. found that adults treated with protein-modified, but often high-energy diets had normal total body protein and BMD both in men and in women, whereas adiposity increased in women.

\section{Session III: Physical activity, exercise, nutrition and body composition}

This session addresses the effects of exercise on body composition; relationships between muscle strength and intra- or extra-cellular water; school physical education, student physical fitness and different physical activities.

Silva et al. indicated that reductions in intra-cellular water increased the risk of losing grip strength in elite judo athletes, along with causing an increase in total body water content. Both the extra-cellular water and intra-cellular water compartments were associated with improvements in strength, independently of fat-free mass changes. Studies also demonstrated energy intake to be a poor predictor of total energy expenditure (TEE), whereas dietary reference intake using estimated physical activity level was reasonably associated with TEE in basketball players during the competitive season. Some studies showed that both aerobic training and resistance training could effectively control body weight, decrease body fat, and increase lean mass and bone density, in several different populations (He et al.). Long-term Tai Chi exercise could enhance the heart function and elasticity of blood vessels in older women (Zhong et al.). It also improved blood-lipid profiles and endocrine function, promoted fat catabolism, and was helpful for fitness and weight reduction (Zhang et al.). Watabe et al. revealed that aerobic exercise could attenuate the loss of skeletal muscle during calorie restriction. Minderico demonstrated that higher cardiorespiratory fitness (CRF) was associated with a lower prevalence of overweight or obesity. CRF also was a better predictor of abdominal fatness than physical activity (Sardinha et al.).

\section{Session IV: Body composition: from infancy to advanced age}

The composition of the body, both in function and in chemical composition, changes naturally with age. Decreases in skeletal muscle mass, organ mass, bone mass, increases in fat stores and changes in the distribution of body fat associated with age have all been well characterized by both cross-sectional and longitudinal studies. Such changes are associated with the increased risks for certain illnesses such as cardiovascular diseases and diabetes. Marked variations of body composition have been found across the early human lifetime. ${ }^{5}$ Sexual differences exist to a small degree at birth, but striking differences develop during the pubertal 
years, which may be largely regulated by endocrine factors. ${ }^{6}$ Women's energy metabolism and weight are influenced by pregnancy. Nutrition and body composition during pregnancy are closely related to the offspring's health. ${ }^{7}$ Menopause is associated with an accelerated loss of fat-free mass and bone mass, and increased central adiposity. Hormone replacement therapy may attenuate these changes. ${ }^{8,9}$

This session includes studies on the different human body composition phenotypes from infancy to the elderly, and their associations with chronic disease risk factors. Kruger et al. found that the prevalence of overweight and obesity was high and increased with age, especially in rural areas in South Africa. Age-specific cutoffs for \%BF may be needed, which is supported by a study conducted by Heo et al. Early life growth patterns could influence programming effects on disease risks in late life. Zhou et al. investigated the association between birth order and adult health conditions, and found that later-born children were on average lighter and had higher blood pressure. An epidemiology study by Hamdouchi et al. found that infant breast-feeding could promote weight loss in mothers, even in the early postpartum period. Using a sample of senior intellectual females, Zhou found that low activity and sedentary behavior would elevate the morbidity of osteoporosis. In addition, several body composition gene studies were also included. Chen et al. found a significant interaction between genes and body composition for the prediction of insulin resistance.

\section{Session V: Development of body composition methodology and its use in clinical practice}

Human body composition measurement is usually used to correlate changes in body components with health risks. Over the past century, in vivo methodology has been developed to quantify body components at the atomic, molecular, cellular, tissue-system and whole-body levels, with continuous improvement and application in clinical practice. This session focuses on the development and comparison of the most commonly used methods, such as bioelectrical impedance analysis (BIA), ultrasound imaging, dilution techniques, air displacement plethysmography, DXA, magnetic resonance imaging and magnetic resonance spectroscopy, and includes the implication of recently developed measurements such as molecular imaging techniques and quantitative magnetic resonance (QMR) in clinical settings.

BIA, among the most non-invasive of all our techniques, is frequently used to estimate body composition based on a $2 \mathrm{C}$ body composition model. Bioimpedance spectroscopy (BIS) allows for the differentiation of total body and extracellular water, which has an added advantage to single-frequency BIA in predicting the fluid balance. ${ }^{1}$ Studies give new insight into the application of BIS in a more accurate way and in a wider area. Zhu et al. explored a 3-C model. Liu et al. discussed the effect of body hydration on the accuracy of body composition by BIS. QMR, a recently developed measurement system for body composition in humans, is also discussed in this session. Bosy-Westphal et al. and Müller et al. investigated the accuracy and validity of QMR.

Numerous abstracts have studied the potential capability of some classic body composition instruments to estimate more comprehensive body composition parameters. Wilson et al. demonstrated a method that calculated total body volume from DXA, which may be more accurate and precise than current methods. Strauss et al. showed that body protein can be reasonably estimated using techniques such as DXA and BIS. Using plasma as a reference, Matias et al. suggested that saliva samples could be accurately used for extra-cellular water assessment.

In conclusion, this supplement contains 11 keynote presentation abstracts, 63 oral presentation abstracts and 131 posters, covering many aspects of body compositionrelated research fields. We believe that the Ninth Symposium will further promote human body composition studies, not only in China but also around the world.

\section{Conflict of interest}

The authors declare no conflict of interest.

\section{References}

1 Lee SY, Gallagher D. Assessment methods in human body composition. Curr Opin Clin Nutr Metab Care 2008; 11: 566-572.

2 Pakiz B, Flatt SW, Bardwell WA, Rock CL, Mills PJ. Effects of a weight loss intervention on body mass, fitness, and inflammatory biomarkers in overweight or obese breast cancer survivors. Int J Behav Med 2011, doi:10.1007/s12529-010-9079-8 (e-pub ahead of print).

3 Bines J, Gradishar WJ. Primary care issues for the breast cancer survivor. Compr Ther 1997; 23: 605-611.

4 Brown BW, Brauner C, Minnotte MC. Noncancer deaths in white adult cancer patients. J Natl Cancer Inst 1993; 85: 979-987.

5 Veldhuis JD, Roemmich JN, Richmond EJ, Rogol AD, Lovejoy JC, Sheffield-Moore $\mathrm{M}$ et al. Endocrine control of body composition in infancy, childhood, and puberty. Endocr Rev 2005; 26: 114-146.

6 Hills AP, Byrne NM. An overview of physical growth and maturation. Med Sport Sci 2010; 55: 1-13.

7 Drake AJ, Reynolds RM. Impact of maternal obesity on offspring obesity and cardiometabolic disease risk. Reproduction 2010; 140: 387-398.

8 Wells G, Tugwell P, Shea B, Guyatt G, Peterson J, Zytaruk N et al. Meta-analyses of therapies for postmenopausal osteoporosis. V. Meta-analysis of the efficacy of hormone replacement therapy in treating and preventing osteoporosis in postmenopausal women. Endocr Rev 2002; 23: 529-539.

9 Chen Z, Bassford T, Green SB, Cauley JA, Jackson RD, LaCroix AZ et al. Postmenopausal hormone therapy and body composition - a substudy of the estrogen plus progestin trial of the Women's Health Initiative. Am J Clin Nutr 2005; 82: 651-656. 